\title{
Generalized Feedback Detection for Spatial Multiplexing Multi-Antenna Systems
}

\author{
Tao Cui and Chintha Tellambura, Senior Member, IEEE
}

\begin{abstract}
We present a unified detection framework for spatial multiplexing multiple-input multiple-output (MIMO) systems by generalizing Heller's classical feedback decoding algorithm for convolutional codes. The resulting generalized feedback detector (GFD) is characterized by three parameters: window size, step size and branch factor. Many existing MIMO detectors are turned out to be special cases of the GFD. Moreover, different parameter choices can provide various performance-complexity tradeoffs. The connection between MIMO detectors and tree search algorithms is also established. To reduce redundant computations in the GFD, a shared computation technique is proposed by using a tree data structure. Using a union bound based analysis of the symbol error rates, the diversity order and signal-to-noise ratio (SNR) gain are derived analytically as functions of the three parameters; for example, the diversity order of the GFD varies between 1 and $N$. The complexity of the GFD varies between those of the maximum-likelihood (ML) detector and the zero-forcing decision feedback detector (ZFDFD). Extensive computer simulation results are also provided.
\end{abstract}

Index Terms-MIMO, feedback decoding, decision feedback detector.

\section{INTRODUCTION}

$\mathbf{S}$ PATIAL multiplexing multiple-input multiple-output (MIMO) systems with rich scattering wireless channels are capable of providing enormous capacity improvements without increasing the bandwidth or transmitted power. A popular system is the Bell-Labs layered space-time (BLAST) architecture [1]. However, the complexity of the maximumlikelihood detector (MLD) increases exponentially with the number of transmit antennas, and researchers have proposed several reduced-complexity suboptimal detectors. The zeroforcing (ZF) decision feedback detector (DFD) with optimal ordering or the V-BLAST detector is proposed in [1]. By using the minimum mean square error (MMSE) principle, the ZFDFD can be extended to the MMSE-DFD [2], offering a compromise between interference suppression and noise enhancement. Nevertheless, since such detectors perform worse than

Manuscript received July 26, 2006; revised June 15, 2007 and November 8, 2007; accepted November 9, 2007. The associate editor coordinating the review of this paper and approving it for publication was J. Andrews. This work has been supported in part by the Natural Sciences and Engineering Research Council of Canada, Informatics Circle of Research Excellence and Alberta Ingenuity Fund. This paper was presented in part at the IEEE Global Telecommunications Conference 2005, Nov. 2005, St. Louis, MO USA.

T. Cui is with the Department of Electrical Engineering, California Institute of Technology, Pasadena, CA 91125 USA (e-mail: taocui@caltech.edu).

C. Tellambura is with the Department of Electrical and Computer Engineering, University of Alberta, Edmonton, AB T6G 2V4, Canada (e-mail: chintha@ece.ualberta.ca).

Digital Object Identifier 10.1109/TWC.2008.060513. the MLD, and since the MLD is complex, recent research has focused on developing high-performance reduced-complexity MIMO detectors.

For example, in [3], a combined detector (ML-DFD) is proposed to detect the first few symbols by using a MLD and the remaining symbols by using a ZF-DFD. In [4], the sphere decoder (SD) is proposed as a MLD, which has low complexity with a high signal-to-noise ratio (SNR). A soft version of SD for coded layered space-time (LST) systems is proposed in [5]. However, with a low SNR or for systems with a large number of transmit antennas, the SD complexity can be high. The Chase decoder for linear block codes has been adopted for MIMO detection in [6]. The performance of the Chase detector varies between those of ML and ZF-BLAST. For instance, the Chase detector achieves a diversity order of 1 or $N$ in an $N \times N$ system, but nothing in between. In [7], the group detector, originally proposed for code-division multipleaccess (CDMA) systems [8], is applied to MIMO detection. The group detector still needs an exhaustive search in each group. A new DFD BLAST algorithm is proposed in [9]. The diversity order of this detector is limited to one, but it can achieve different SNR gains. Moreover, linear detectors using the relaxation approach have also been developed. For CDMA systems, a generalized MMSE (GMMSE) detector is proposed [10], where the binary phase shift-keying (BPSK) vectors are relaxed so that they lie inside the smallest hypersphere containing the unit hypercube. In [11], a tighter relaxation is used to develop a constrained least squares (CLS) detector for orthogonal frequency division multiplexing (OFDM) / spatial division multiple access (SDMA) systems employing unitary signal constellations. A similar detection problem also arises for systems over both scalar and vector frequency selective channels [12]-[14].

This paper develops a unified detection framework for spatial multiplexing systems such as the V-BLAST. We reformulate the MIMO detection problem as a set of overlapping subdetection problems; the feedback decoder of Heller [15] for convolutional codes is then extended to the new generalized feedback MIMO detector (GFD) with three characteristic parameters: window size, step size and branch factor. Although the subdetection problems can, of course, be solved by exhaustive search, we propose using Schnorr and Euchner's SD (SESD) for improved computational efficiency [16]. With different parameter values, appropriate channel matrix ordering and subdetectors, the GFD provides various performance-complexity tradeoffs and also yields many well-known algorithms such as the ZF-BLAST [1], the SD [4], the combined ML and ZF- 
DFD [3], the B-Chase detector [6], and the group detector [7], [8] as special cases. Moreover, all these detectors can be explained as tree search algorithms. If linear subdetectors are employed, the GFD also generalizes the L-Chase detector in [6]. A reduced-complexity shared computation technique is also proposed, ensuring the GFD complexity varies between those of the ZF-DFD and the MLD. Using the union bound (UB) on the symbol error probability, we analytically derive the diversity order and SNR gain of the GFD. For example, the diversity order of the GFD varies between 1 and $N$. Extensive simulation results are also provided.

This paper is organized as follows. Section II describes the system model and reviews feedback decoding. Section III develops the new generalized feedback detector and the computation-sharing technique. Section IV analyzes the diversity order and SNR gain of the GFD. The simulation results and conclusions are given in Sections $\mathrm{V}$ and VI.

Notation: Bold symbols denote matrices or vectors. $(\cdot)^{T},(\cdot)^{H}$ and $(\cdot)^{*}$ denote the transpose, conjugate transpose and conjugate, respectively. $(\cdot)^{\dagger}$ denotes the pseudo-inverse. $\|(\cdot)\|^{2}$ is the 2-norm of $(\cdot) . E\{(\cdot)\}$ is the expectation of $(\cdot)$. $P[(\cdot)]$ is the probability of $(\cdot)$. The set of all complex $K \times 1$ vectors is denoted by $\mathcal{C}^{K}$. A circularly complex Gaussian variable with mean $\mu$ and variance $\sigma^{2}$ is $z \sim \mathcal{C N}\left(\mu, \sigma^{2}\right) . A^{c}$ denotes the complement event of $A$. The $N \times N$ identity matrix is denoted by $\mathbf{I}_{N}$. The Matlab notation is used to represent the elements of a vector.

\section{System Model And FEedBACK DeCOding}

\section{A. Problem formulation}

We consider a spatial multiplexing $n$ transmit antenna and $m$ receive antenna MIMO system. The input bit stream is demultiplexed into $n$ independent streams which are modulated by the same complex constellation $\mathcal{Q}$. We consider a rich scattering flat-fading MIMO channel. The baseband received signals at a given discrete time instant can thus be written as

$$
\mathbf{r}=\mathbf{H x}+\mathbf{n}
$$

where $\mathbf{x}=\left[x_{1}, \ldots, x_{n}\right]^{T}, x_{i} \in \mathcal{Q}$ is the transmitted signal vector, $\mathbf{r}=\left[r_{1}, \ldots, r_{m}\right]^{T}, r_{i} \in \mathcal{C}$ is the received signal vector, $\mathbf{H}=\left[h_{i, j}\right] \in \mathcal{C}^{m \times n}$ is the channel matrix, and $\mathbf{n}=\left[n_{1}, \ldots, n_{m}\right]^{T}, n_{i} \in \mathcal{C N}\left(0, \sigma_{n}^{2}\right)$ is an additive white Gaussian noise (AWGN) vector, and $n_{i}$ 's are independent and identically distributed (i.i.d.), as are the components of $\mathbf{H}$; i.e., $h_{i, j} \sim \mathcal{C N}(0,1)$.

The channel is known to the receiver, and the MLD is given by

$$
\hat{\mathbf{x}}=\underset{\mathbf{x} \in \mathcal{Q}^{n}}{\arg \min }\|\mathbf{r}-\mathbf{H} \mathbf{x}\|^{2} .
$$

Due to the discrete alphabet $\mathcal{Q}$, linear detectors such as leastsquares detectors generally do not give the optimal solution. If $\mathcal{Q}$ is a subset of the integer set $\mathcal{Z}$, (2) is known as the closest vector problem (CVP) in lattice theory and is known to be NP-hard [17], [18]. The exhaustive search for (2) has a complexity exponential in $n$. We assume that the number of transmit antennas is less than or equal to the number of receive antennas $(n \leq m)$. If $n>m$, the system becomes rank-deficient, and our proposed GFD may be extended to this case via the method in [19].

For practical purposes, the MLD problem (2) is often transformed by column reordering so that initial detector decisions will be as error free as possible. Column reordering can be by using V-BLAST or other ordering schemes [2], [4], and the resulting channel matrix is $\mathbf{G}=\mathbf{H \Pi}$, where $\boldsymbol{\Pi}$ is the column permutation matrix. Let the $\mathrm{QR}$ factorization of $\mathbf{G}=\mathbf{Q R}$, where $\mathbf{R}$ is an $n \times n$ upper-triangular matrix, and $\mathbf{Q}$ is unitary. Eq. (2) is equivalent to

$$
\hat{\mathbf{x}}=\underset{\mathbf{x} \in \mathcal{Q}^{n}}{\arg \min }\|\mathbf{y}-\mathbf{R} \mathbf{x}\|^{2},
$$

where $\mathbf{y}=\mathbf{Q}^{H} \mathbf{r}$. Eq. (3) is the basis for our GFD and other detectors [1], [3], [4], [6].

\section{B. Feedback decoding}

Three classical decoding algorithms are known for convolutional codes [20]: Viterbi decoding, sequential decoding and feedback decoding. Heller's feedback decoder [15] sacrifices performance in exchange for complexity reduction. However, to the best of our knowledge, has not been applied yet to MIMO detection. Our generalization of this decoder not only provides a unified framework for describing virtually all existing MIMO detectors, but also enables new ones.

In binary systems, Heller's feedback decoder makes a hard decision on $(j+1)$-th bit based on metrics computed from stage $(j+1)$ to stage $(j+w)$, where $w$ is a positive integer. The decoder then proceeds to the $(j+2)$-th bit, and metrics computed from stage $(j+2)$ to stage $(j+1+w)$ are used to make a hard decision. The same procedure is repeated for all the bits. A smaller $w$ requires less complexity and memory, but results in a larger performance loss.

We next show how Heller's decoder can be adapted for MIMO detection.

\section{Generalized FeEdBACK Detector}

\section{A. Basic algorithm with one parameter}

The GFD is now developed for (3). For simplicity, a single parameter case is treated first. The first parameter to be considered is window size $w$, which is a preselected positive integer. The cost metric to be minimized in (3) can be written as

$$
\sum_{i=1}^{n}\left(\left|y_{i}-\sum_{j=i}^{n} r_{i, j} x_{j}\right|^{2}\right),
$$

where $r_{i, j}$ is the $(i, j)$-th entry of matrix $\mathbf{R}$. Since $\mathbf{R}$ is uppertriangular, the $i$-th term in (4) depends only on $x_{i}, \ldots, x_{n}$, $1 \leq i \leq n$. The detector starts from $x_{n}$. In the $k$-th step, using the hard decisions $\hat{x}_{i}, n-k+1<i \leq n$, the detector makes a decision on $x_{n-k+1}$ based on metrics computed from $y_{n-k-w+2}$ to $y_{n-k+1}$. We first determine the subvector $\mathbf{x}^{(k)}=$ $\left[x_{n-k-w+2}, \ldots, x_{n-k+1}\right]^{T}$ by using (5).

In principle, an exhaustive search can solve (5), and the resulting $\hat{\mathbf{x}}^{(k)}$ contains $w$ hard decisions. However, we discard $w-1$ of them in order to improve the overall error rate. 


$$
\hat{\mathbf{x}}^{(k)}=\underset{\mathbf{x}^{(k)} \in \mathcal{Q}^{w}}{\arg \min } \sum_{i=n-k-w+2}^{n-k+1}\left(\left|y_{i}-\sum_{j=i}^{n-k+1} r_{i, j} x_{j}-\sum_{j=n-k+2}^{n} r_{i, j} \hat{x}_{j}\right|^{2}\right)
$$

Consequently, the hard decision on $x_{n-k+1}$ is made to be $\hat{x}_{n-k+1}=\hat{\mathbf{x}}^{(k)}(w)$, where $\hat{\mathbf{x}}^{(k)}(w)$ denotes the $w$-th element in $\hat{\mathbf{x}}^{(k)}$. The same procedure is performed for $x_{k-1}$ based on the metric computed from $y_{k-w}$ to $y_{k-1}$. The GFD can also be considered as a sliding window detector of size $w$, making a decision on each $x_{k}$ based on the minimum metric within the window. Note that this version of the GFD which has only one parameter (namely, the window size) is essentially identical to Heller's original feedback decoder.

At this point, it is worth mentioning several special cases of the GFD. When the window size is unity $(w=1)$, GFD reduces to the ZF-DFD or the V-BLAST detector. When the window size increases to $n$, GFD becomes equivalent to the MLD. When the window size varies between 1 and $n$, the performance of the GFD is between those of the MLD and the ZF-DFD.

\section{B. Two additional parameters}

The above GFD in now generalized in two steps. First, the detection process is divided into $K$ stages, and the window size can (possibly) change in each stage. So the constant parameter $w$ is replaced by the set $\left\{w_{1}, w_{2}, \ldots, w_{K}\right\}$. Second, the number of hard decisions made in the $k$ th stage is set to $s_{k}$ (step size). That is, instead of making a single decision at each stage, hard decisions $\hat{x}_{l_{k}-s_{k}+1}, \ldots, \hat{x}_{l_{k}}$ are made at the $k$-th stage, where $l_{k}=n-\sum_{k^{\prime}=1}^{k-1} s_{k^{\prime}}$ and $s_{k^{\prime}}$ is the step size at the $k^{\prime}$-th stage. The subvector $\mathbf{x}^{(k)}=\left[x_{l_{k}-w_{k}+1}, \ldots, x_{l_{k}}\right]^{T}$ is detected by using

$\hat{\mathbf{x}}^{(k)}=\underset{\mathbf{x}^{(k)} \in \mathcal{Q}^{w_{k}}}{\arg \min } \sum_{i=l_{k}-w_{k}+1}^{l_{k}}\left(\left|y_{i}-\sum_{j=i}^{l_{k}} r_{i, j} x_{j}-\sum_{j=l_{k}+1}^{n} r_{i, j} \hat{x}_{j}\right|^{2}\right)$,

where $w_{k}$ is the window size in the $k$-th stage. Hard decisions are made as $\left[\hat{x}_{l_{k}-s_{k}+1}, \ldots, \hat{x}_{l_{k}}\right]^{T}=\hat{\mathbf{x}}^{(k)}\left(w_{k}-s_{k}+1: w_{k}\right)$. Proceeding to the $(k+1)$-th stage, the window size is changed to $w_{k+1}$. The group of symbols $x_{l_{k+1}-s_{k+1}+1}, \ldots, x_{l_{k+1}}$ is decided in this stage. If the detector has $K$ stages, $\sum_{k=1}^{K} s_{k}=$ $n$. The basic algorithm with different $w_{k}$ and $s_{k}$ is illustrated in Fig. 1, where the shadowed regions correspond to hard decisions.

In (6), $s_{k}$ hard decisions are made at the $k$-th stage. However, if this number is large, the overall error performance degrades rapidly. To overcome this problem, we suggest one more generalization of the GFD. The key idea is to generate a list of candidates for $\mathrm{x}^{(k)}$ that minimize (6), instead of returning the best candidate. The size of the list is $b_{k}$, named the branch factor. For example, for $k=1$, generate $b_{1}$ best candidates minimizing (6) and store the corresponding different postfix vectors $\mathbf{x}^{(1)}\left(w_{1}-s_{1}+1: w_{1}\right)$ in a list $\mathcal{L}_{1}$ with $q_{1}$ elements. Note that $1 \leq q_{1} \leq b_{1}$ because there may be repeated postfix vectors. For each $\left[\tilde{x}_{n-s_{1}+1}, \ldots, \tilde{x}_{n}\right]^{T}$ in $\mathcal{L}_{1}$, the candidate list $\mathcal{L}_{2}$ in the second stage is generated. The

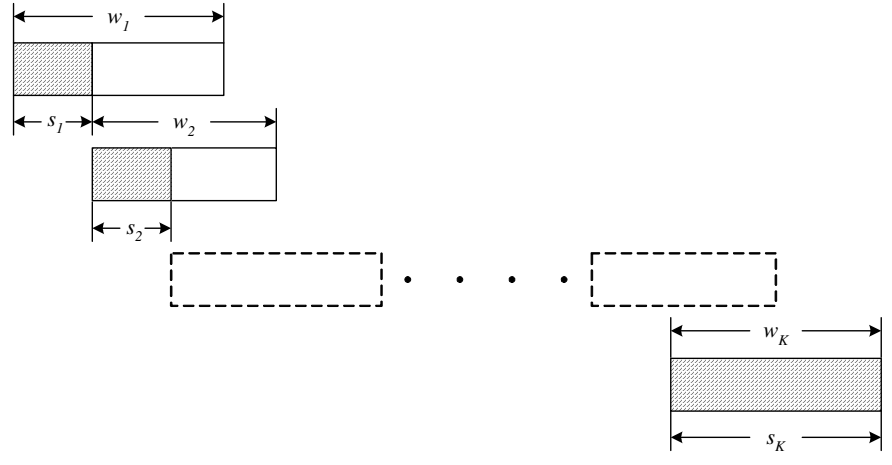

Fig. 1. Basic algorithm illustration of the GFD with different $w_{k}$ and $s_{k}$.

same procedure is performed iteratively. Note that $b_{k}$ may vary in different stages. This algorithm in fact shrinks the search space from $\mathcal{Q}^{n}$ to a new space $\mathcal{A}(w, s, b)$, which depends on the window size, step size and branch factor. The detection problem is then

$$
\hat{\mathbf{x}}=\underset{\mathbf{x} \in \mathcal{A}(w, s, b)}{\arg \min }\|\mathbf{y}-\mathbf{R} \mathbf{x}\|^{2} .
$$

If $b_{k}=1$ for $k=1, \ldots, K,(7)$ reduces to the detector in the second case. If $b_{k}=1, s_{k}=1$ and $w_{k}=w$, (7) reduces to the original feedback detection algorithm. Note in the $K$-th stage, $b_{K}=1$ and $s_{K}=w_{K}$ as imposed by the end state condition.

Both (6) and (7) entail an exhaustive search in a reduced space. The SD [4] efficiently solves (6) and (7) without the exhaustive search. In particular, Schnorr and Euchner's SD (SESD) [16] removes the dependence on the initial radius and hence may be preferred. When $b_{k} \neq 0$, the list sphere decoder (LSD) [21] may also be used to create the candidate list.

Clearly, if $w_{k}=1, s_{k}=1$ and $b_{k}=1$, the GFD reduces to the ZF-DFD [1], and if $w_{k}=n, s_{k}=n$ and $b_{k}=1$, the GFD is equivalent to solving the MLD via the SD [4]. When $w_{1}=p, w_{k}=1(k>1), s_{1}=p, s_{k}=1(k>1)$ and $b_{k}=1$, the GFD reduces to a combination of the MLD and the ZF-DFD [3]. When $w_{k}=1, s_{k}=1, b_{1}=q$ and $b_{k}=1$ $(k>1)$, the GFD becomes the B-Chase detector proposed in [6] by using a different column permutation matrix. When $w_{k}=s_{k}, b_{k}=1$ and MLD is used as the subdetector, the GFD becomes the group detector [7], [8]. Table I summarizes the relationship between these detectors and the GFD. Different detectors are also characterized by the subdetector used in each stage. For example, all these detectors use an exhaustive search for subdetection problems. Clearly, all these exhaustive searches can be replaced by SDs, as in our GFD framework.

\section{Remarks:}

- The proposed GFD uses an SD for the subproblem (6) in each stage. Of course, the complexity further reduces if a suboptimal detector such as LS, MMSE or GMMSE [10], 
TABLE I

SPECIAL CASES OF THE GENERALIZED FEEDBACK DETECTOR.

\begin{tabular}{c||c|c|c}
\hline Detector & Window size $w_{k}$ & Step size $s_{k}$ & Branch factor $b_{k}$ \\
\hline \hline ZF-DFD & $w_{k}=1$ & $s_{k}=1$ & $b_{k}=1$ \\
\hline SD & $w_{k}=n$ & $s_{k}=n$ & $b_{k}=1$ \\
\hline ML-DFD & $\begin{array}{c}w_{1}=p, \\
w_{k}=1, k>1\end{array}$ & $\begin{array}{c}s_{1}=p, \\
s_{k}=1, k>1\end{array}$ & $b_{k}=1$ \\
\hline B-Chase & $w_{k}=1$ & $s_{k}=1$ & $\begin{array}{c}b_{1}=q, \\
b_{k}=1, k>1\end{array}$ \\
\hline Group detector & $w_{k}=s_{k}$ & $w_{k}=s_{k}$ & $b_{k}=1$ \\
\hline
\end{tabular}

or CLS [11] is used in each stage. More generally, the GFD may flexibly employ $K_{1}$ SDs and $K-K_{1}$ linear detectors. For example, as shown in Section IV, since reliable detection of the first few stages is critical to the overall system performance, they can employ the SD, with remaining stages using linear detectors to reduce the overall complexity. For example, if $w_{1}=s_{1}=1$, $w_{2}=s_{2}=n-1, b_{1}=q$, and $b_{2}=1$, and an SD is used in the first stage, and a linear detector is used in the second stage, our GFD reduces to the L-Chase detector in [6]. Our GFD thus generalizes [6] and provide flexible complexity and performance tradeoffs.

- Our proposed generalizations involving step size and branch factor parameter can be adapted in Heller's feedback decoder as well. That would result in a new class of decoders for convolutional codes. With appropriate $w, s, b$, the GFD can also be used for candidate-list generation in the MIMO system capacity-achieving algorithm in [21].

- The detector in [9] is a special case of our GFD with unit step size. The diversity order of the detector [9] is only one, but it can achieve different SNR gains.

\section{Tree interpretation}

Many MIMO detectors [1], [3], [4], [6] are equivalent to a tree search. The detectors traverse through a $|\mathcal{Q}|$-ary tree of $n$ levels, where $|\mathcal{Q}|$ is the cardinality of constellation $\mathcal{Q}$. Except for the leaf nodes, $|\mathcal{Q}|$ branches stem from each node, and each branch is labeled by an element from $\mathcal{Q}$. A node at the $k$-th level is assigned a metric,

$$
m_{k}\left(\mathbf{x}_{k}\right)=\left|y_{n-k+1}-\sum_{j=n-k+1}^{n} r_{n-k+1, j} x_{j}\right|^{2},
$$

where $\mathbf{x}_{k}=\left[x_{n-k+1}, \ldots, x_{n}\right]^{T}$ are the symbols labeling the path from the root to this node. The accumulated path metric associated with path $\mathbf{x}_{k}$ is thus defined as

$$
c\left(\mathbf{x}_{k}\right)=\sum_{i=n-k+1}^{n} m_{i}\left(\mathbf{x}_{i}\right)=\sum_{i=n-k+1}^{n}\left|y_{i}-\sum_{j=i}^{n} r_{i, j} x_{j}\right|^{2} .
$$

The MLD performs an exhaustive tree search by computing the accumulated path metric (9) for all possible tree paths from the root to leaf nodes. The path with a minimum accumulated metric is output as the ML solution.
The complexity of the MLD is reduced by the SD [4], which explores only a subset of the tree by discarding nodes that cannot lead to the MLD solution. In the SESD, the processing order depends on the accumulated metric. Thus, the child node with a minimum accumulated metric is expanded first. This ordering ensures that whenever the SESD reaches a leaf node, its path cost is as small as possible, and thus the optimal path is found as rapidly as possible.

Our proposed GFD expands only a set of partial trees instead of the full tree in the MLD. At the $k$-th stage, for $x_{l_{k}}, \ldots, x_{n}$, the GFD searches through a $w_{k}$ level tree stemming from $x_{l_{k}}$. The $q_{k}$ best partial paths from the root to the leaf nodes of the partial tree with a minimum accumulated metric are chosen, where $q_{k}$ is the size of the candidate list. The $x_{l_{k}-1}, \ldots, x_{l_{k}-s_{k}}$ symbols are chosen corresponding to each partial path. The window shifts $s_{k}$ symbols, and a partial tree is searched again. In fact, our GFD forms a reduced tree with $K$ levels. At the $k$-th level, there are $q_{k}$ branches from a node, which is assigned a metric,

$$
\tilde{m}_{k}\left(\mathbf{x}_{k}\right)=\sum_{i=l_{k}-s_{k}}^{l_{k}-1}\left|y_{i}-\sum_{j=i}^{n} r_{i, j} x_{j}\right|^{2},
$$

where $\mathbf{x}_{k}=\left[x_{l_{k}-s_{k}}, \ldots, x_{n}\right]^{T}$ are the symbols labels on the path from the root to this node. The SD and DFDs can be applied to the reduced tree. If $b_{k}=1$, the GFD reduces to ZF-DFD, or the SD can be used to search through the new tree.

Fig. 2 illustrates the GFD on a tree with 4 levels (i.e., $n=4)$ and binary phase shift keying (BPSK). The left side is the full tree expanded by an exhaustive search. The GFD parameters are $w_{1}=3, s_{1}=2$ and $b_{1}=2$. An exhaustive search or LSD is used to traverse the first partial tree; $\left[x_{4}, x_{3}, x_{2}\right]^{T}=\left\{[+1,+1,-1]^{T},[-1,+1,+1]^{T}\right\}$ are the $b_{1}$ subvectors that make the partial accumulated metric from $x_{4}$ to $x_{2}$ a minimum. For each subvector, $\left[x_{4}, x_{3}\right]^{T}$ is stored in a list: $\mathcal{L}_{1}=\left\{[+1,+1]^{T},[-1,+1]^{T}\right\}$. The $b_{1}$ elements form $b_{1}$ branches in the new tree in the right side of Fig. 1. In the second stage, the window is shifted by $s_{1}=2$ to the second rectangle, and the window size, step size and branch factor are changed to $s_{2}=w_{2}=2$ and $b_{2}=1$. Therefore, the original 4-level tree with 16 leaf nodes is reduced to a 2-level tree with 2 leaf nodes by using the GFD. The SD or the SESD can be used to find the path with a minimum accumulated metric in the reduced tree. The corresponding path of the new tree is the output sequence. The ZF-DFD expands only a single branch tree (see Fig. 2 (c)). 


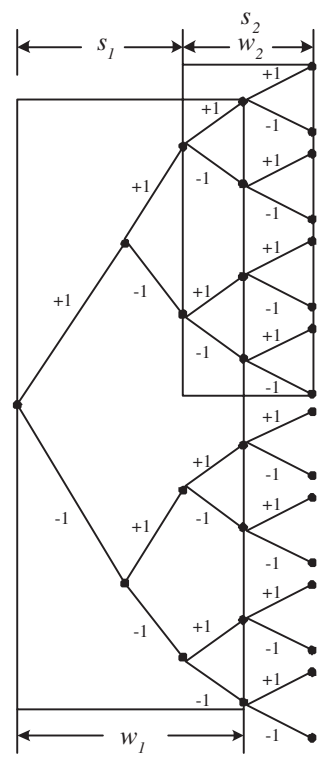

(a)

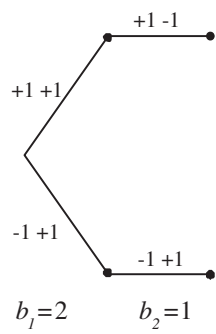

(b)

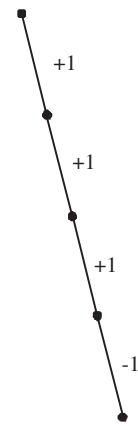

(c)
Fig. 2. Comparison of exhaustive search, GFD and ZF-DFD in tree representation for $n=m=4$ and BPSK.

The B-Chase detector [6] with list size $q$ and a ZFDFD subdetector forms a 2-level tree with $q$ leaf nodes; an exhaustive search will find the minimum path metric in the tree.

Our GFD forms different trees with different parameters $w_{k}, s_{k}$ and $b_{k}$. Different parameter sets provide different performances and complexities (Section IV).

\section{Computation-sharing technique}

The simple case with $b_{k}=1$ is considered first. The discussion can be readily extended to other cases. A detailed complexity analysis is given in Section IV, Subsection B.

The basic GFD constitutes $K-1$ SDs and a ZF-DFD on a $w_{K}$ level tree. The complexities of the SD and the DFD for an $n \times n$ system are denoted by $C_{\mathrm{SD}}(n)$ and $C_{\mathrm{DFD}}(n)$, respectively. Thus, the complexity of the GFD can be written as

$$
C_{\mathrm{GFD}}(n)=\sum_{k=1}^{K-1} C_{\mathrm{SD}}\left(w_{k}\right)+C_{\mathrm{DFD}}\left(w_{K}\right),
$$

where the last term is due to the use of the DFD in the last stage.

In the high $\mathrm{SNR}, C_{\mathrm{SD}}\left(w_{k}\right) \simeq w_{k}^{2}$ [17], [18]. In this region, it is possible that the complexity of the GFD exceeds that of the SD. To eliminate this possibility, we introduce a shared computation technique that eliminates redundant computations in the basic GFD. In the $k$-th stage, if $w_{k} \neq s_{k}$, there will be $w_{k}-s_{k}$ symbols' overlap between the $k$-th window and the $(k+1)$-th window. The basic GFD uses two SDs in the two windows. However, due to the overlap, some nodes of the partial tree in the $(k+1)$-th stage have been visited by the sphere decoder in the $k$-th stage. We thus propose to store all the metrics for the visited nodes in the $k$-th SD to avoid repeated computations. The tree is a suitable data structure for the visited nodes storage since the SD forms a tree during the search. When using the SD for the $(k+1)$-th partial tree, the SD traverses the search tree and the storage tree at the same time. Only the subtree corresponding to the selected $\left[x_{l_{k}-s_{k}+1}, \ldots, x_{l_{k}}\right]^{T}$ is kept, and the other subtrees are pruned. If the node has not been visited as indicated in the storage tree, the branch metric is computed, and the node is added to the storage tree.

\section{Performance And Complexity Analysis}

\section{A. Performance analysis}

The case of the equal number of transmit and receive antennas is considered. In the GFD, we assume $w_{k}=w$, $s_{k}=s$ and $b_{k}=1$. The analysis can be easily extended to $n \neq m$ and general GFDs. To make the performance analysis tractable, a system without channel matrix ordering is analyzed. Since any reasonable channel matrix ordering improves the detector performance, our analysis gives an upper bound on the error rate and hence a lower bound on the performance.

To guide the reader, it is worthwhile to mention the basic theme of the whole performance analysis. First of all, the diversity order and SNR gain are meaningful only in the high SNR region. In this region, a detector's error probability, say, $P_{s}$, has the following approximate behavior as a function of the SNR:

$$
P_{s} \approx[\alpha S N R]^{-\beta}
$$

where $\alpha$ is the SNR gain and $\beta$ is the diversity order. To find $P_{s}$ in this format, the conditional probabilities are expressed in terms of the Q-function and averaged over the appropriate distribution.

From [22], the squared norm of the entries of uppertriangular matrix $\mathbf{R}$ have $\chi^{2}$ distribution with different degrees of freedom, specifically, $\left|r_{i, i}\right|^{2} \sim \chi^{2}(2 i)$, for $i=1, \ldots, n$ and $\left|r_{i, j}\right|^{2} \sim \chi^{2}(2)$, for $j>i$, where $\chi^{2}(k)$ denotes the chisquared distribution with $k$ degrees of freedom. Let $\mathbf{v}=\mathbf{Q n}$, where $\mathbf{Q}$ is defined as in (3). As $\mathbf{Q}$ is unitary, $\mathbf{v}$ is also an i.i.d. complex Gaussian vector with mean zero and variance $\sigma_{n}^{2}$

In the first stage of the GFD, ML decoding is performed with $\mathbf{y}_{1}=\mathbf{R}_{1} \mathbf{x}_{1}+\mathbf{v}_{1}$ to detect $\mathbf{x}_{1}=\left[x_{n-w+1}, \ldots, x_{n}\right]^{T}$, where $\mathbf{y}_{1}=\left[y_{n-w+1}, \ldots, y_{n}\right]^{T}, \mathbf{v}_{1}=\left[v_{n-w+1}, \ldots, v_{n}\right]^{T}$ and $\mathbf{R}_{1}=\mathbf{R}(n-w+1: n, n-w+1: n)$. Denote the block error event in the $k$-th stage by $E_{k}$. The union bound for the block error probability of $\mathbf{x}_{1}, P_{b}\left(E_{1}\right)$, is given by

$$
P_{b}\left(E_{1}\right) \leq \underset{\mathbf{R}_{1}}{E} \underset{\mathbf{x}_{1}^{(1)}}{E}\left[\sum_{\hat{\mathbf{x}}_{1}, \mathbf{x}_{1}^{(2)}} P\left(\hat{\mathbf{x}}_{1}=\mathbf{x}_{1}^{(2)} \mid \mathbf{x}_{1}^{(1)}, \mathbf{R}_{1}\right)\right],
$$

where $\mathbf{x}_{1}^{(2)}$ are all the possible vectors other than $\mathbf{x}_{1}^{(1)}$, and

$$
P\left(\hat{\mathbf{x}}_{1}=\mathbf{x}_{1}^{(2)} \mid \mathbf{x}_{1}^{(1)}, \mathbf{R}_{1}\right)=Q\left(\sqrt{\left\|\mathbf{R}_{1}\left(\mathbf{x}_{1}^{(2)}-\mathbf{x}_{1}^{(1)}\right)\right\|^{2} / 2 \sigma_{n}^{2}}\right),
$$


where $Q(\cdot)$ is the Q-function. Let $\mathbf{H}_{2}$ be a $w \times w$ matrix with each entry complex Gaussian and its $\mathrm{QR}$ decomposition be $\mathbf{H}_{2}=\mathbf{Q}_{2} \mathbf{R}_{2}$. Since $\mathbf{R}_{2}$ has the same distribution as $\mathbf{R}_{1},\left\|\mathbf{R}_{2}\left(\mathbf{x}_{1}^{(2)}-\mathbf{x}_{1}^{(1)}\right)\right\|^{2}$ has the same distribution as $\left\|\mathbf{R}_{1}\left(\mathbf{x}_{1}^{(2)}-\mathbf{x}_{1}^{(1)}\right)\right\|^{2}$. Since $\mathbf{Q}_{2}$ is unitary, the norm can be rearranged as

$$
\begin{array}{r}
\left\|\mathbf{R}_{2}\left(\mathbf{x}_{1}^{(2)}-\mathbf{x}_{1}^{(1)}\right)\right\|^{2}=\left\|\mathbf{Q}_{2} \mathbf{R}_{2}\left(\mathbf{x}_{1}^{(2)}-\mathbf{x}_{1}^{(1)}\right)\right\|^{2} \\
=\left\|\mathbf{H}_{2}\left(\mathbf{x}_{1}^{(2)}-\mathbf{x}_{1}^{(1)}\right)\right\|^{2}=a_{1}\left\|\mathbf{x}_{1}^{(2)}-\mathbf{x}_{1}^{(1)}\right\|^{2},
\end{array}
$$

where $a_{1} \sim \chi^{2}(2 w)$, and (14) follows from the fact that a linear combination of independent Gaussian variables is also Gaussian. Eq (13) thus reduces to

$$
P\left(\hat{\mathbf{x}}_{1}=\mathbf{x}_{1}^{(2)} \mid \mathbf{x}_{1}^{(1)}, a_{1}\right)=Q\left(\sqrt{a_{1}\left\|\mathbf{x}_{1}^{(2)}-\mathbf{x}_{1}^{(1)}\right\|^{2} / 2 \sigma_{n}^{2}}\right) .
$$

By using the Chernoff bound for the Q-function [20], $P_{b}\left(\mathbf{x}_{1}\right)$ can be bounded as

$$
\begin{aligned}
P_{b}\left(E_{1}\right) & \leq \underset{a_{1}}{\underset{\mathbf{x}_{1}^{(1)}}{E}}\left[\sum_{\mathbf{x}_{1}^{(2)}} \exp \left(-a_{1}\left\|\mathbf{x}_{1}^{(2)}-\mathbf{x}_{1}^{(1)}\right\|^{2} / 4 \sigma_{n}^{2}\right)\right] \\
& =\underset{\mathbf{x}_{1}^{(1)}}{E} \sum_{\mathbf{x}_{1}^{(2)}} \frac{1}{\left(1+\frac{\left\|\mathbf{x}_{1}^{(2)}-\mathbf{x}_{1}^{(1)}\right\|^{2}}{4 \sigma_{n}^{2}}\right)^{w}} \\
& \leq\left(\frac{|\mathcal{Q}|}{1+d_{\min }^{2} / 4 \sigma_{n}^{2}}\right)^{w}
\end{aligned}
$$

where $d_{\min }$ is the minimum distance between $\mathbf{x}_{1}^{(1)}$ and $\mathbf{x}_{1}^{(2)}$. In the high SNR, a block error results from a single symbol error in $\mathbf{x}_{1}$. Therefore, the bound for the symbol error probability is $P_{s}\left(x_{i}\right)=P_{b}\left(E_{1}\right) / w, i=n-s+1, \ldots, n$, and the bound for the block error probability of $\tilde{\mathbf{x}}_{1}=\left[x_{n-s+1}, \ldots, x_{n}\right]^{T}$ is $P_{b}\left(\tilde{E}_{1}\right)=P_{b}\left(E_{1}\right) s / w$, where $\tilde{E}_{1}$ is the block error event in $\tilde{\mathbf{x}}_{1}$. In the $k$-th stage, let $\tilde{\mathbf{x}}_{k}=\left[x_{n-(k-1) s-w+1}, \ldots, x_{n-(k-1) s}\right]^{T}$, and denote $\tilde{E}_{k}$ as the block error event in $\tilde{\mathbf{x}}_{k}$. The probability for the event $E_{k}$ conditional on $\left(\cup_{i=1}^{k-1} \tilde{E}_{i}\right)^{c}$ is equal to the error probability of ML decoding of $\mathbf{x}_{k}$ from $\mathbf{y}_{k}=\mathbf{R}_{k} \mathbf{x}_{k}+\mathbf{v}_{k}$, where $\quad \mathbf{x}_{k}=\left[x_{n-(k-1) s-w+1}, \ldots, x_{n-(k-1) s}\right]^{T}$, $\mathbf{y}_{k}=\left[y_{n-(k-1) s-w+1}, \ldots, y_{n-(k-1) s}\right]^{T}, \quad \mathbf{v}_{k}=$ $\left[v_{n-(k-1) s-w+1}, \ldots, v_{n-(k-1) s}\right]^{T}$, and $\mathbf{R}_{k}=\mathbf{R}(n-(k-$ 1) $s-w+1: n-(k-1) s, n-(k-1) s-w+1: n-(k-1) s)$. $\mathbf{y}_{k}$ is obtained by cancelling the contribution of $\tilde{\mathbf{x}}_{i}$, $i=1, \ldots, k-1$, from $\mathbf{y}$. The union bound for $P\left(E_{k} \mid\left(\cup_{i=1}^{k-1} \tilde{E}_{i}\right)^{c}\right)$ can thus obtained as

$P\left(E_{k} \mid\left(\cup_{i=1}^{k-1} \tilde{E}_{i}\right)^{c}\right) \leq \underset{\mathbf{x}_{k}^{(1)}}{E}\left[\sum_{\mathbf{x}_{k}^{(2)}} P\left(\hat{\mathbf{x}}_{k}=\mathbf{x}_{k}^{(2)} \mid \mathbf{x}_{k}^{(1)}, \mathbf{R}_{k}\right)\right]$,

where $\mathbf{x}_{k}^{(2)}$ are all the possible vectors other than $\mathbf{x}_{k}^{(1)}$, and

$$
P\left(\hat{\mathbf{x}}_{k}=\mathbf{x}_{k}^{(2)} \mid \mathbf{x}_{k}^{(1)}, \mathbf{R}_{k}\right)=Q\left(\sqrt{\left\|\mathbf{R}_{k}\left(\mathbf{x}_{k}^{(2)}-\mathbf{x}_{k}^{(1)}\right)\right\|^{2} / 2 \sigma_{n}^{2}}\right) .
$$

Let $\mathbf{H}_{2}$ be a $(k-1) s+w \times(k-1) s+w$ matrix with each entry complex Gaussian and its $\mathrm{QR}$ decomposition be $\mathbf{H}_{2}=\mathbf{Q}_{2} \mathbf{R}_{2}$. Define two new vectors $\tilde{\mathbf{x}}_{k}^{(1)}=\left[\left(\mathbf{x}_{k}^{(1)}\right)^{H}, \mathbf{0}_{(k-1) s \times 1}\right]^{T}$ and $\tilde{\mathbf{x}}_{k}^{(2)}=\left[\left(\mathbf{x}_{k}^{(2)}\right)^{H}, \mathbf{0}_{(k-1) s \times 1}\right]^{T}$. Since $\mathbf{R}_{2}((k-1) s+1:(k-$ $1) s+w,(k-1) s+1:(k-1) s+w)$ has the same distribution as $\mathbf{R}_{k}$, and $\left\|\mathbf{R}_{2}\left(\tilde{\mathbf{x}}_{k}^{(2)}-\tilde{\mathbf{x}}_{k}^{(1)}\right)\right\|^{2}$ has the same distribution as $\left\|\mathbf{R}_{k}\left(\mathbf{x}_{k}^{(2)}-\mathbf{x}_{k}^{(1)}\right)\right\|^{2}$. Since $\mathbf{Q}_{2}$ is unitary, similar to (14), we have

$$
\begin{array}{r}
\left\|\mathbf{R}_{2}\left(\tilde{\mathbf{x}}_{k}^{(2)}-\tilde{\mathbf{x}}_{k}^{(1)}\right)\right\|^{2}=\left\|\mathbf{Q}_{2} \mathbf{R}_{2}\left(\tilde{\mathbf{x}}_{k}^{(2)}-\tilde{\mathbf{x}}_{k}^{(1)}\right)\right\|^{2} \\
=\left\|\mathbf{H}_{2}\left(\tilde{\mathbf{x}}_{k}^{(2)}-\tilde{\mathbf{x}}_{k}^{(1)}\right)\right\|^{2}=a_{k}\left\|\mathbf{x}_{1}^{(2)}-\mathbf{x}_{1}^{(1)}\right\|^{2},
\end{array}
$$

where $a_{k}$ is a random variable, and $a_{k} \sim \chi^{2}(2(k-1) s+2 w)$. We have

$$
P\left(E_{k} \mid\left(\cup_{i=1}^{k-1} \tilde{E}_{i}\right)^{c}\right) \leq\left(\frac{|\mathcal{Q}|}{1+d_{\min }^{2} / 4 \sigma_{n}^{2}}\right)^{w+(k-1) s},
$$

which is similar to (16). Using the total probability theorem [22] and noting that the events $\left(\cup_{i=1}^{k-1} \tilde{E}_{i}\right)^{c}, \tilde{E}_{k-1} \cap$ $\left(\cup_{i=1}^{k-2} \tilde{E}_{i}\right)^{c}, \ldots, \tilde{E}_{1}$ are exclusive, we have $(21)$, where the inequality $(a)$ is obtained by assuming that given an error event on $\tilde{E}_{i}, 1 \leq i<k$, the event $E_{k}$ has probability 1 . The equality $(b)$ comes from the Bayes' theorem [22]. By using the chain rule for conditional probability, $(b)$ can be computed in closed form. However, it is complicated and does not provide an insight into the diversity order and SNR gain of the detector. Note that with a high SNR, $P\left(\cap_{i=1}^{j-1} \tilde{E}_{i}^{c}\right)$ is close to 1 . We can thus further simplify $P_{b}\left(E_{k}\right)$ as

$$
P_{b}\left(E_{k}\right) \leq P\left(E_{k} \mid\left(\cup_{i=1}^{k-1} \tilde{E}_{i}\right)^{c}\right)+\sum_{j=1}^{k-1} P\left(\tilde{E}_{j} \mid \cap_{i=1}^{j-1} \tilde{E}_{i}^{c}\right) .
$$

Similarly, with a high SNR, we have

$$
P\left(\tilde{E}_{j} \mid \cap_{i=1}^{j-1} \tilde{E}_{i}^{c}\right)=\frac{s}{w} P_{b}\left(E_{j} \mid \cap_{i=1}^{j-1} \tilde{E}_{i}^{c}\right)=\frac{s}{w} P_{b}\left(E_{1}\right) \epsilon^{j-1},
$$

where $\epsilon=\left(\frac{|\mathcal{Q}|}{1+d_{\min }^{2} / 4 \sigma_{n}^{2}}\right)^{s}$. Substituting (23) into (22), we can obtain

$$
\begin{aligned}
P_{b}\left(E_{k}\right) & \leq P_{b}\left(E_{1}\right) \epsilon^{k-1}+\frac{s}{w} P_{b}\left(E_{1}\right)\left(1+\epsilon+\cdots+\epsilon^{k-2}\right) \\
& \leq \frac{s}{w} P_{b}\left(E_{1}\right)(1+\delta),
\end{aligned}
$$

where $\delta=\sum_{i=1}^{k-2} \epsilon^{i}+\frac{w}{s} \epsilon^{k-1}$. With a high SNR, where $\delta$ is a small positive number, $(s / w) P_{b}\left(E_{1}\right)$ dominates (24). Therefore, the average symbol error probability can be approximated as

$$
\begin{aligned}
P_{s} & =\frac{1}{n}\left(\frac{s}{w} P_{b}\left(E_{1}\right)+\frac{s}{w} \sum_{k=2}^{K-1} P_{b}\left(E_{k}\right)+P_{b}\left(E_{K}\right)\right) \\
& \simeq \frac{1}{n}\left(\frac{2 s}{w}+(K-2) \frac{s^{2}}{w^{2}}\right)\left(\frac{|\mathcal{Q}|}{1+d_{\min }^{2} / 4 \sigma_{n}^{2}}\right)^{w} .
\end{aligned}
$$




$$
\begin{aligned}
P_{b}\left(E_{k}\right) & =P\left(E_{k} \mid \tilde{E}_{1}\right) P\left(\tilde{E}_{1}\right)+P\left(E_{k} \mid \tilde{E}_{2} \cap \tilde{E}_{1}^{c}\right) P\left(\tilde{E}_{2} \cap \tilde{E}_{1}^{c}\right)+\cdots \\
& +P\left(E_{k} \mid \tilde{E}_{k-1} \cap\left(\cup_{i=1}^{k-2} \tilde{E}_{i}\right)^{c}\right) P\left(\tilde{E}_{k-1} \cap\left(\cup_{i=1}^{k-2} \tilde{E}_{i}\right)^{c}\right) \\
& +P\left(E_{k} \mid\left(\cup_{i=1}^{k-1} \tilde{E}_{i}\right)^{c}\right) P\left(\left(\cup_{i=1}^{k-1} \tilde{E}_{i}\right)^{c}\right) \\
& \stackrel{a}{\leq} P\left(\tilde{E}_{1}\right)+P\left(\tilde{E}_{2} \cap \tilde{E}_{1}^{c}\right)+\cdots+P\left(E_{k} \mid\left(\cup_{i=1}^{k-1} \tilde{E}_{i}\right)^{c}\right) P\left(\left(\cup_{i=1}^{k-1} \tilde{E}_{i}\right)^{c}\right) \\
& \stackrel{b}{=} \sum_{j=1}^{k-1} P\left(\tilde{E}_{j} \mid \cap_{i=1}^{j-1} \tilde{E}_{i}^{c}\right) P\left(\cap_{i=1}^{j-1} \tilde{E}_{i}^{c}\right)+P\left(E_{k} \mid\left(\cup_{i=1}^{k-1} \tilde{E}_{i}\right)^{c}\right) P\left(\cap_{i=1}^{k-1} \tilde{E}_{i}^{c}\right),
\end{aligned}
$$

Eq. (25) shows that the GFD has a diversity order $w$ and that different values for the step size and window size provide different SNR gains. The union bound (25) is loose, and the actual SNR gain may be different. From further performance analysis, we can show that if the $w_{k}$ 's are different, the performance of the GFD is limited by the first window size, and that the diversity order is $w_{1}$. The simulations show that if $b_{k}<|\mathcal{Q}|$, different $b_{k}$ 's achieve different SNR gains, while choosing $b_{k}=|\mathcal{Q}|$ attains a higher diversity order. However, the case of $b_{k}>1$ is not amenable to exact analysis.

\section{B. Computational complexity analysis}

The computational complexity may be measured in terms of the best, worst, or average-case complexity [23]. The average complexity of the SD derived in [17], [18] is exponential with $n$ but low with a high SNR. The SD has been realized on VLSI circuits in [24], [25]. For VLSI circuits implementation, the throughput is limited by the worst-case complexity. We next derive the worst-case complexity of the GFD. The average complexity of the GFD is difficult to derive analytically and is determined via simulation (Section V).

We assume that elements in both $\mathbf{R}$ and $\mathcal{Q}$ are complex. A single complex addition and multiplication take 2 and 7 flops, respectively. In the first stage, since $\mathbf{R}$ is upper triangular, the number of flops is

$$
\begin{aligned}
C_{1}\left(w_{1}\right) & =\sum_{i=1}^{w_{1}}|\mathcal{Q}|^{i}\left[9\left(w_{1}+1-i\right)+5\right]=5 \frac{|\mathcal{Q}|^{w_{1}+1}-|\mathcal{Q}|}{|\mathcal{Q}|-1} \\
& +9 \frac{|\mathcal{Q}|^{w_{1}+2}-\left(w_{1}+1\right)|\mathcal{Q}|^{2}+w_{1}|\mathcal{Q}|}{(|\mathcal{Q}|-1)^{2}}
\end{aligned}
$$

For the $k$-th stage $(1<k<K)$, the accumulated path metrics for all the combinations of the first $t_{k}=w_{k-1}-s_{k-1}$ variables have been computed in the $(k-1)$-th stage. Due to the computation sharing, the number of flops in the $k$-th stage is

$C_{k}\left(w_{k}\right)=\sum_{i=t_{k}+1}^{w_{k}}|\mathcal{Q}|^{i}\left[9\left(w_{1}+1-i\right)+5\right]=C_{1}\left(w_{k}\right)-C_{1}\left(t_{k}\right)$.

In the $K$-th stage, a hard decision is applied, and the number of flops is $C_{K}\left(w_{K}\right)=\sum_{i=1}^{w_{K}}(9 i+3)=\frac{9 w_{K}\left(w_{K}+1\right)}{2}+3 w_{K}$. Therefore, the total number of flops for the GFD is given by

$$
C_{\mathrm{GFD}}=\sum_{k=1}^{K} \prod_{i=1}^{k-1} b_{i} C_{k}\left(w_{k}\right)
$$

For the special case of $w_{k}=w, s_{k}=s$ and $b_{k}=1$, we have

$$
\begin{aligned}
C_{\mathrm{GFD}} & =C_{1}(w)+(K-2)\left(C_{1}(w)-C_{1}(w-s)\right)+C_{K}\left(w_{K}\right) \\
& \leq 2 C_{1}(w)+(K-2)\left(C_{1}(w)-C_{1}(w-s)\right) \\
& =2 C_{1}(w)+\left(\frac{n-w_{K}}{s}-2\right)\left(C_{1}(w)-C_{1}(w-s)\right),
\end{aligned}
$$

where we have used the fact that $w \geq w_{K}$ and $C_{K}(w) \leq$ $C_{1}(w)$. Interestingly, (26) and (29) indicate that the worstcase complexity of the GFD is exponential in window size. Hence, increasing the diversity order of the GFD also increases the worst-case complexity exponent. When both $n$ and $w$ are large, $C_{1}(w)$ scales as $|\mathcal{Q}|^{w}$ and the second term in the last equality in (29) scales as $\frac{n|\mathcal{Q}|^{w}\left(1-|\mathcal{Q}|^{-s}\right)}{s}$, which is a decreasing function in $s$ when $|\mathcal{Q}|>1$ and $s \geq 1$. Thus, (29) suggests that decreasing the step size increases the complexity but does not change the exponent. This finding agrees with that of the performance analysis, that changing the step size changes only the SNR gain.

\section{Simulation Results}

The GFD is simulated for an uncoded MIMO system with 8 transmit and 8 receive antennas over a flat Rayleigh fading channel. Comparisons are made with the V-BLAST and the SD in terms of both performance and complexity. $\operatorname{GFD}[\alpha, \beta, \gamma]$ denotes a GFD with a window size $\alpha$, step size $\beta$, and branch factor $\gamma$. The V-BLAST column reordering is applied in all the detectors. The MATLAB V5.3 command "flops" is used to count the number of flops. Only the flops of the search algorithm are counted, and the preprocessing stage is ignored.

Figs. 3 and 4 show the BER and average number of flops for different detectors in a BPSK modulated system. In the GFD, we set $w_{k}=w, s_{k}=s$ and $b_{k}=1$. The SESD [16] is used with an infinite initial radius. The effect of window size and step size is investigated. Clearly, with different window sizes, different diversity orders are achieved (Fig. 3). $\operatorname{GFD}[2,1,1]$ has a $3-\mathrm{dB}$ gain over $\mathrm{GFD}[2,2,1]$ at $\mathrm{BER}=10^{-4}$. GFD $[4,1,1]$ performs $0.5 \mathrm{~dB}$ better than GFD $[4,2,1]$ and 1.5 $\mathrm{dB}$ better than GFD $[4,4,1]$ at $\mathrm{BER}=10^{-5}$. Therefore, the SNR gain diminishes with the increasing step size. With different parameter settings, the GFD also has different complexity levels (Fig. 4). The complexity of the GFD varies between those of the V-BLAST and the SD. In high SNRs, the average 


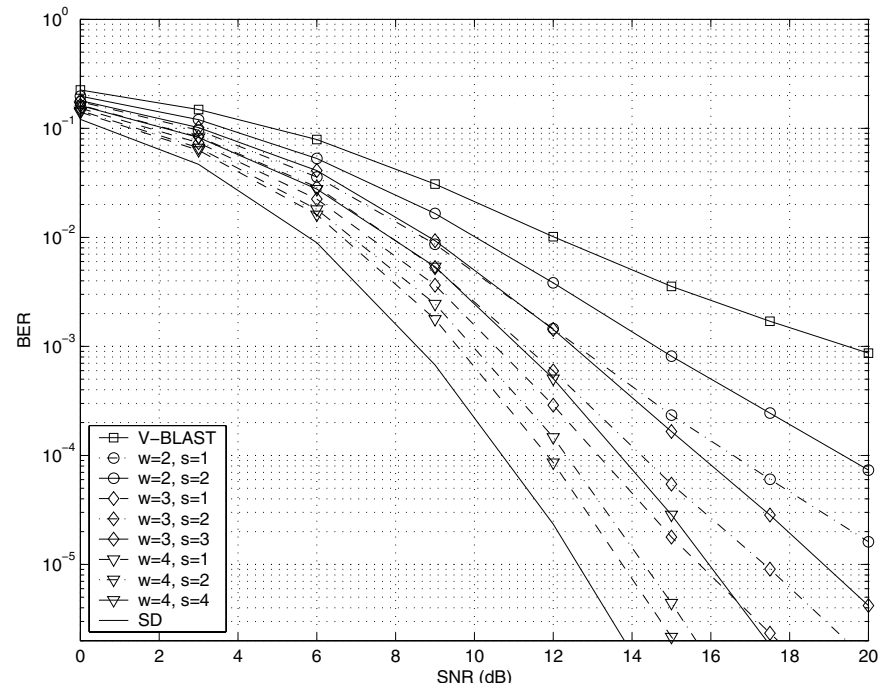

Fig. 3. BER comparison of different detectors in an $8 \times 8$ BPSK MIMO system. In the GFD, we set $w_{k}=w, s_{k}=s$ and $b_{k}=1$.

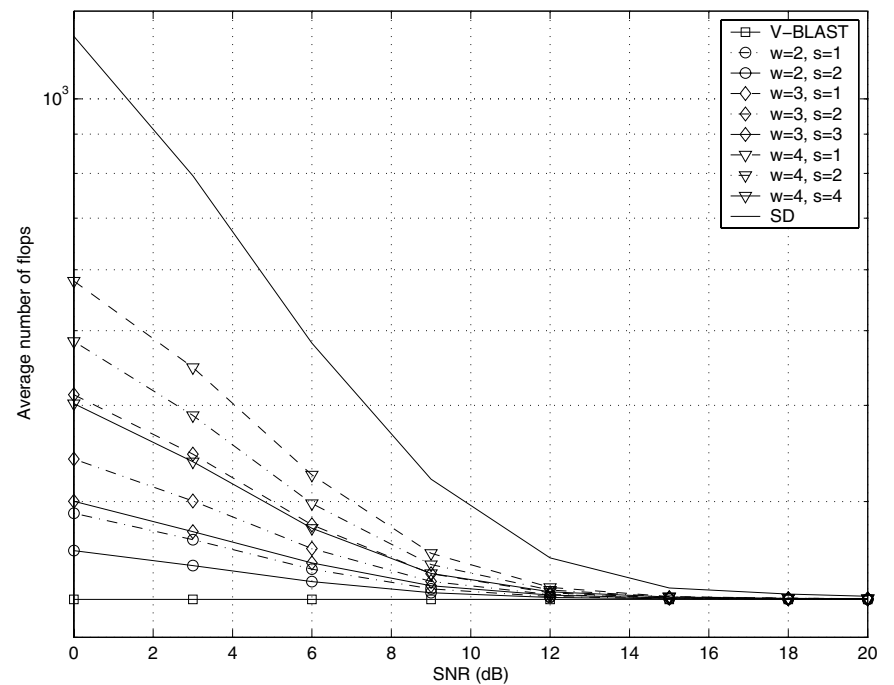

Fig. 4. Average complexity comparison of different detectors in an $8 \times 8$ BPSK MIMO system. In the GFD, we set $w_{k}=w, s_{k}=s$ and $b_{k}=1$.

complexity is roughly the same for all these detectors. With a low SNR, the smaller the step size, the higher complexity should be. As well, the average complexity is decreased by increasing the window size with the same step size.

Figs. 5 and 6 compare the BER and average number of flops for different detectors in a BPSK system. In the GFD, we also set $w_{k}=w, s_{k}=s$ but $b_{k} \geq 1$. Instead of using the SESD [16], the initial radius is chosen to be proportional to the noise variance as in [17]. If no solution is found with the initial radius, the radius repeatedly doubled until a solution is found. This method of radius selection is the best method because the SESD may expand redundant branches in the reduced tree of the GFD, and each branch needs a high complexity to increase the window size and step size. With the same window size, the diversity order of the GFD is the same, but a different SNR gain is achieved. GFD[2,2,2] has a 5$\mathrm{dB}$ gain over $\mathrm{GFD}[2,2,1]$ at $\mathrm{BER}=10^{-3}$. The performance

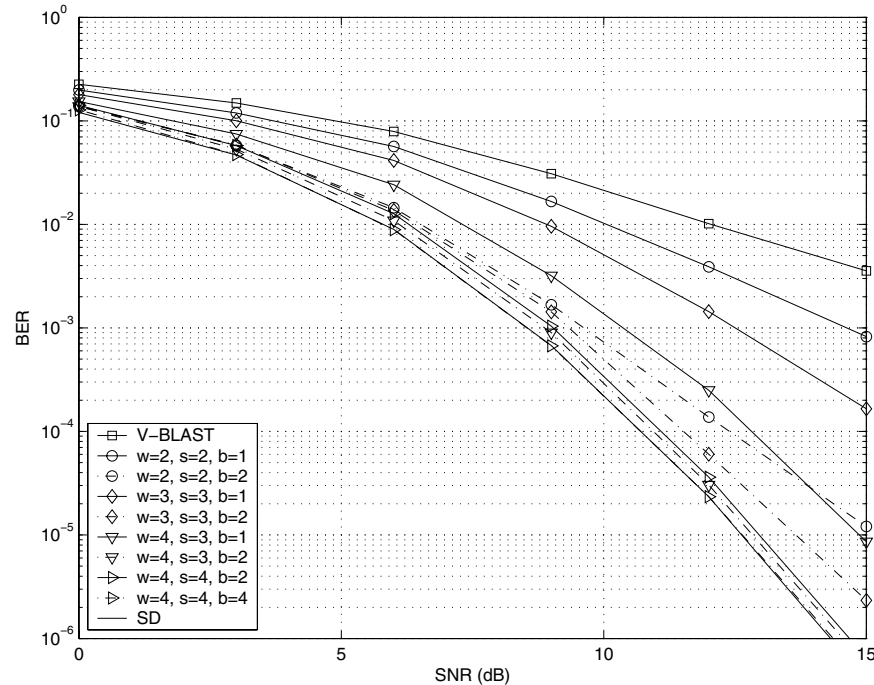

Fig. 5. BER comparison of different detectors in an $8 \times 8$ BPSK MIMO system. In the GFD, we set $w_{k}=w, s_{k}=s$ but $b_{k} \geq 1$.

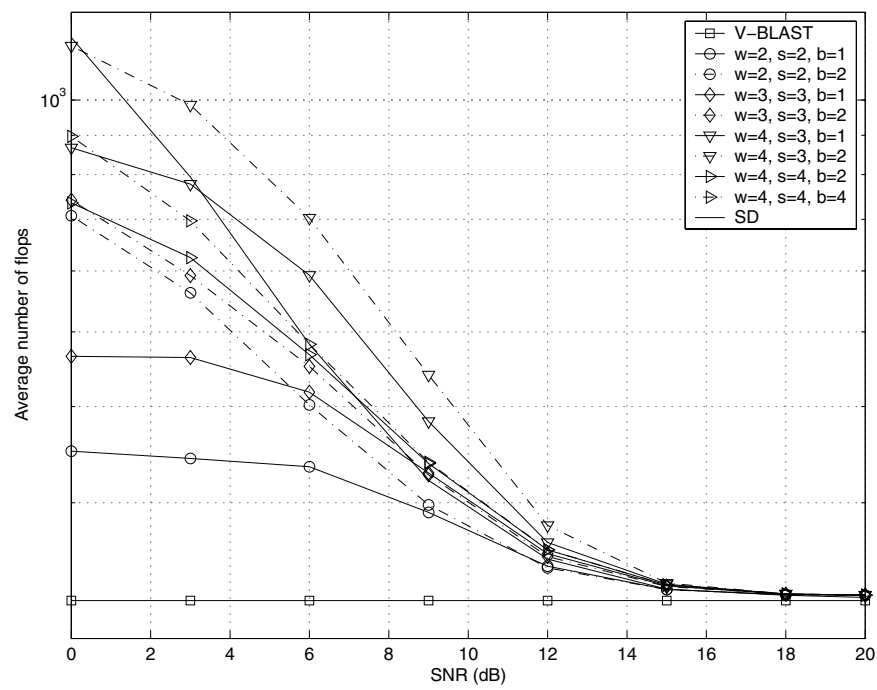

Fig. 6. Average complexity comparison of different detectors in an $8 \times 8$ BPSK MIMO system. In the GFD, we set $w_{k}=w, s_{k}=s$ but $b_{k} \geq 1$.

gap between GFD[3,3,1] and GFD[2,2,2] reduces to $3 \mathrm{~dB}$ at $\mathrm{BER}=10^{-3}$. GFD $[4,4,4]$ has only a $0.5 \mathrm{~dB}$ gain over $\operatorname{GFD}[4,4,2]$ at $\mathrm{BER}=10^{-4}$. GFD $[4,4,4]$ performs close to the SD. If the window size is increased, the SNR gain achieved by increasing the branch factor also diminishes, but with the same window size, the SNR gain achieved by increasing the branch factor is larger than that achieved by decreasing the step size. With a different window size, step size and branch factor, the average complexity is also different. With a high SNR, the complexity of all the detectors is the same. However, with a low SNR, due to the use of the noise variance's initial radius, the average complexity approaches the worstcase complexity. The complexity of GFD $[4,3,2]$ is higher than that of GFD $[4,4,2]$ because the former forms a 3-level tree while the latter forms only a 2-level tree. With a low SNR, GFD $[4,4,4]$ has a lower complexity than the SD but almost the same performance. 


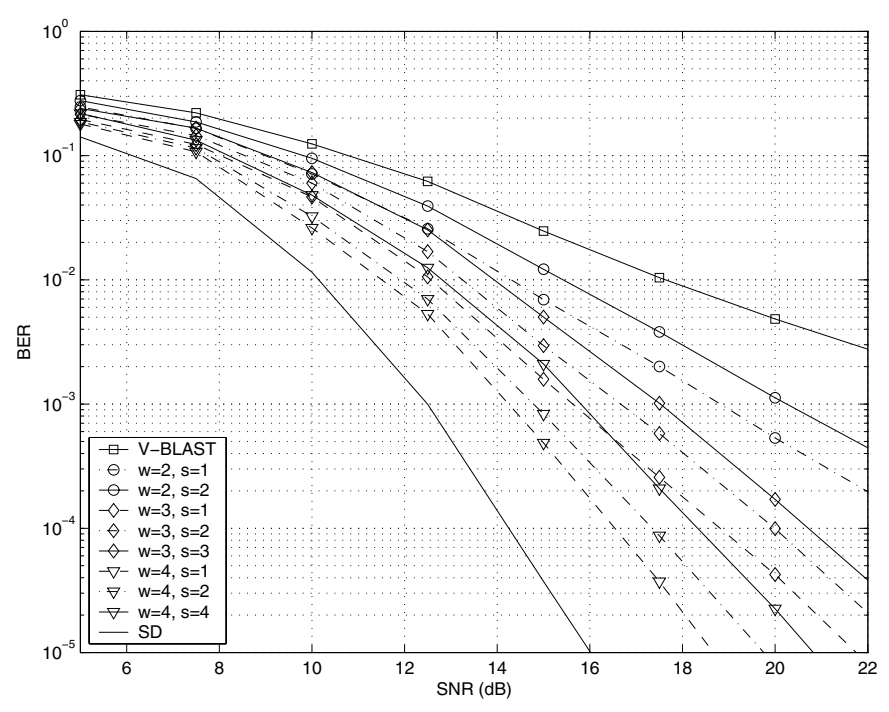

Fig. 7. BER comparison of different detectors in an $8 \times 8$ 4QAM MIMO system. In the GFD, we set $w_{k}=w, s_{k}=s$ and $b_{k}=1$.

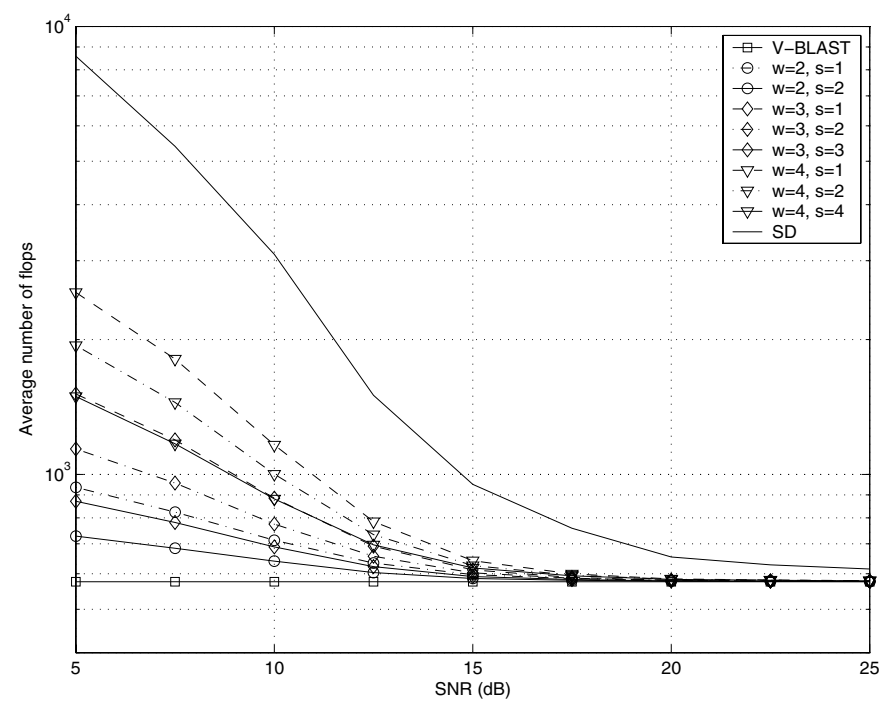

Fig. 8. Average complexity comparison of different detectors in an $8 \times 8$ 4QAM MIMO system. In the GFD, we set $w_{k}=w, s_{k}=s$ and $b_{k}=1$.

In Figs. 7 and 8, we compare different detectors in a 4quadrature amplitude modulation (QAM) system with Gray mapping and $w_{k}=w, s_{k}=s$ and $b_{k}=1$. The SESD [16] with an infinite initial radius is used. Both the performance and complexity results are similar to those of BPSK systems. The SNR gain achieved by decreasing the step size reduces with the constellation size. For example, for the BPSK system, GFD $[2,1,1]$ has a $3-\mathrm{dB}$ gain over GFD $[2,2,1]$ at $\mathrm{BER}=10^{-4}$. However, the SNR gain is reduced to $2.4 \mathrm{~dB}$ for $4 \mathrm{QAM}$ at $\mathrm{BER}=10^{-3}$.

Finally, in Figs. 9 and 10, the BER and average number of flops for different detectors in a 4QAM system with Gray mapping is shown. The SESD is not used as in Figs. 5 and 6. We fix the window size and step size, and observe the effect of the branch factor. The performance of the B-Chase detector [6] is also evaluated. GFD[2,2,2] has a 4-dB gain over $\mathrm{GFD}[2,2,1]$ at a BER of $10^{-3}$. GFD[3,3,2] has a $3-\mathrm{dB}$ gain

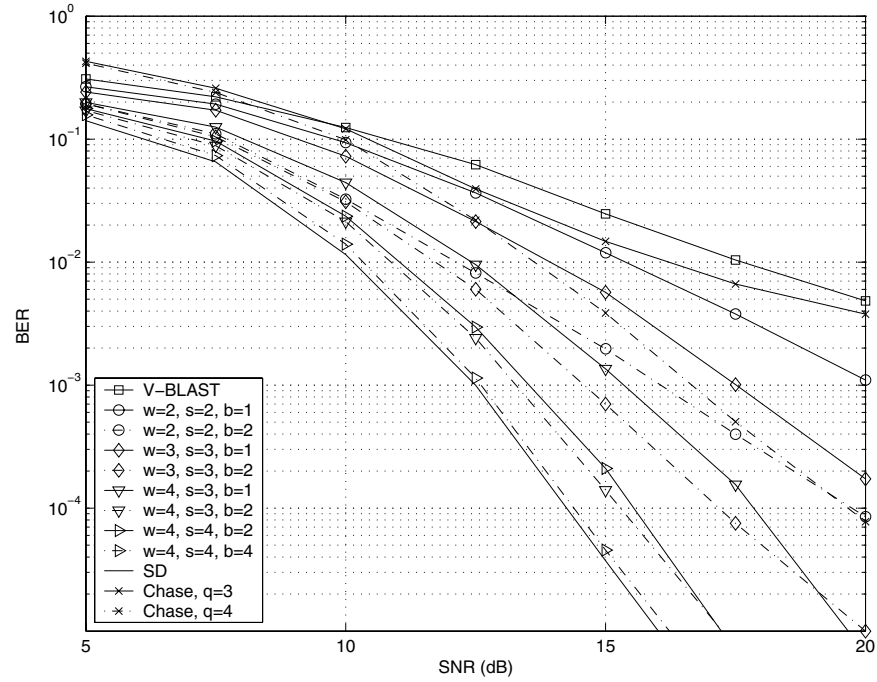

Fig. 9. BER comparison of different detectors in an $8 \times 8$ 4QAM MIMO system. In the GFD, we set $w_{k}=w, s_{k}=s$ but $b_{k} \geq 1$.

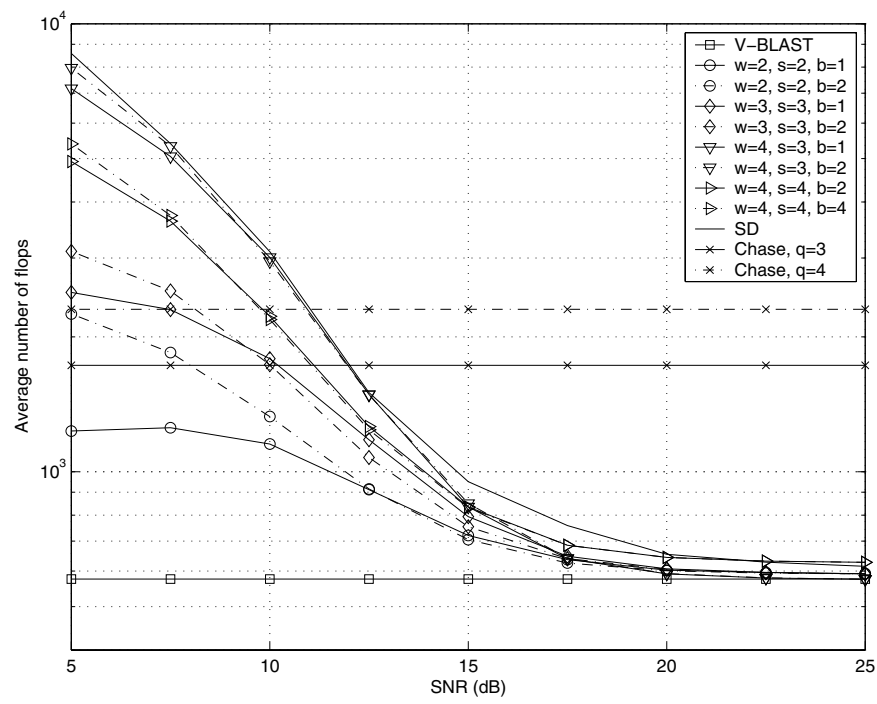

Fig. 10. Average complexity comparison of different detectors in an $8 \times 8$ 4QAM MIMO system. In the GFD, we set $w_{k}=w, s_{k}=s$ but $b_{k} \geq 1$.

over GFD[3,3,1] at BER $=2 \times 10^{-4}$. The gain is reduced to $2 \mathrm{~dB}$ with $w=3$ and $s=3$ at $\mathrm{BER}=10^{-4}$, but all the performance gaps become constant with a high SNR. GFD $[4,4,4]$ performs close to the SD. The SNR gain achieved by increasing the branch factor diminishes with an increase of the constellation size. The B-Chase detector with $q=3$ performs better than the V-BLAST but much worse than the other detectors. The BChase detector with $q=4$ has a significant performance gain over that with $q=3$ and performs identically as GFD[2,2,2] at $\mathrm{SNR}=20 \mathrm{~dB}$. We cannot determine the diversity order of the B-Chase detector with $q=4$ from the SNR region in Fig. 9, but the ordering in [6] indeed provides a significant performance gain. The complexity results are similar to those in Fig. 6. We do not count the complexity of preprocessing for the B-Chase detector. The complexity of all the detectors decreases with an increase of the SNR. GFD $[4,4,4]$ has a lower complexity than the SD but achieves almost the same 
performance. GFD $2,2,2]$ has a better performance than the B-Chase detector with $q=4$, but with lower complexity, especially in high SNRs. The GFD appears to achieve a better performance-complexity tradeoff than the Chase detector [6].

\section{CONCLUSION}

We have proposed a unified MIMO signal detection framework by generalizing the classical feedback decoding. The proposed GFD employs a set of sub-detectors, which can be chosen depending on performance and complexity constraints. The GFD performance and complexity vary between those of SD and V-BLAST. By deriving the union bound for the symbol error probability of the GFD, we show that the GFD achieves an arbitrary diversity order between 1 and $n$ (the number of antennas) and different SNR gains. We also establish the connection between MIMO detectors and the tree search algorithms. Moreover, a computation-sharing technique is proposed to further reduce the complexity. Our proposed GFD provides a flexible performance-complexity tradeoff for MIMO detection and can be extended to applications such as decoding convolutional codes and equalization in MIMO frequency-selective channels [13]. Interesting further work could include performance analysis of the GFD when channel matrix ordering is applied.

\section{ACKNOWLEDGMENT}

The authors wish to thank the anonymous reviewers for their careful reading of the manuscript and critical comments, which greatly improved this paper.

\section{REFERENCES}

[1] G. D. Golden, G. J. Foschini, R. A. Valenzuela, and P. W. Wolniansky, "Detection algorithm and initial laboratory results using the V-BLAST space-time communication architecture," Electron. Lett., vol. 35, no. 1, pp. 14-15, Jan. 1999.

[2] A. Benjebbour, H. Murata, and S. Yoshida, "Comparison of ordered successive receivers for space-time transmission," in Proc. VTC 2001 Fall, vol. 4, Oct. 2001, pp. 2053-2057.

[3] W.-J. Choi, R. Negi, and J. M. Cioffi, "Combined ML and DFE decoding for the V-BLAST system," in Proc. ICC 2000, vol. 3, June 2000, pp. $1243-1248$.

[4] M. O. Damen, E. Gamal, and G. Caire, "On maximum-likelihood detection and the search for the closest lattice point," IEEE Trans. Inf. Theory, vol. 49, no. 10, pp. 2389-2402, Oct. 2003.

[5] R. Wang and G. B. Giannakis, "Approaching MIMO channel capacity with soft detection based on hard sphere decoding," IEEE Trans. Commun., vol. 54, no. 4, pp. 587-590, Apr. 2006.

[6] D. W. Waters and J. R. Barry, "The chase family of detection algorithms for multiple-input multiple-output channels," in Proc. GLOBECOM '04, vol. 4, Nov. 2004, pp. 2635-2639.

[7] X. Li, H. Huang, A. Lozano, and G. Foschini, "Reduced-complexity detection algorithms for systems using multi-element arrays," in Proc. IEEE GLOBECOM, vol. 2, Nov 2000, pp. 1072-1076.

[8] M. K. Varanasi, "Group detection for synchronous gaussian codedivision multiple access," IEEE Trans. Inf. Theory, vol. 41, no. 4, pp. 1083-1096, July 1995.

[9] A. Bhargave, R. de Figueiredo, and T. Eltoft, "A detection algorithm for the V-BLAST system," in Proc. IEEE GLOBECOM, vol. 1, Nov 2001, pp. 494-498.

[10] A. Yener, R. D. Yates, and S. Ulukus, "CDMA multiuser detection: a nonlinear programming approach," IEEE Trans. Commun., vol. 50, no. 6, pp. 1016-1024, June 2002.

[11] S. Thoen, L. Deneire, L. V. der Perre, M. Engels, and H. D. Man, "Constrained least squares detector for OFDM/SDMA-based wireless networks," IEEE Trans. Wireless Commun., vol. 2, no. 1, pp. 129-140, Jan. 2003.
[12] R. Schober, L.-J. Lampe, W. Gerstacker, and S. Pasupathy, "Modulation diversity for frequency-selective fading channels," IEEE Trans. Inf. Theory, vol. 49, no. 9, pp. 2268-2276, Sept. 2003.

[13] R. Schober, W. Gerstacker, and L.-J. Lampe, "Performance analysis and design of STBCs for frequency-selective fading channels," IEEE Trans. Wireless Commun., vol. 3, no. 3, pp. 734-744, May 2004.

[14] H. Gerstacker, R. Schober, and A. Lampe, "Receivers with widely linear processing for frequency-selective channels," IEEE Trans. Commun., vol. 51, no. 9, pp. 1512-1523, Sept. 2003.

[15] J. A. Heller, "Feedback decoding of convolutional codes," in Advances in Communications Systems, A. J. Viterbi, Ed., vol. 4. NY: Academic Press, 1975, pp. 261-278.

[16] C. P. Schnorr and M. Euchner, "Lattice basis reduction: Improved practical algorithms and solving subset sum problems," Math. Programming, vol. 66, pp. 181-191, 1994.

[17] B. Hassibi and H. Vikalo, "On the expected complexity of integer leastsquares problems," in Proc. IEEE ICASSP, vol. 2, 2002, pp. 1497-1500.

[18] J. Jalden and B. Ottersten, "On the complexity of sphere decoding in digital communications," IEEE Trans. Signal Process., vol. 53, no. 4. pp. 1474-1484, April 2005

[19] T. Cui and C. Tellambura, "An efficient generalized sphere decoder for rank-deficient MIMO systems," IEEE Commun. Lett., vol. 9, no. 5, pp. 423-425, May 2005.

[20] J. G. Proakis, Digital Communications, 4th ed. McGraw-Hill, 2001.

[21] B. Hochwald and S. ten Brink, "Achieving near-capacity on a multipleantenna channel," IEEE Trans. Commun., vol. 51, no. 3, pp. 389-399, Mar. 2003.

[22] A. Papoulis and S. Pillai, Probability, Random Variables and Stochastic Processes. McGraw-Hill Education, Dec. 2001.

[23] S. S. Skiena, The Algorithm Design Manual, 1st ed. Springer-Verlag, 1997.

[24] D. Garrett, L. Davis, S. Brink, B. Hochwald, and G. Knagge, "Silicon complexity for maximum likelihood MIMO detection using spherical decoding," IEEE J. Solid-State Circuits, vol. 39, no. 9, pp. 1544-1552, Sept. 2004

[25] A. Burg, M. Borgmann, C. Simon, M. Wenk, M. Zellweger, and W. Fichtner, "Performance tradeoffs in the VLSI implementation of the sphere decoding algorithm," in Proc. IEE $3 G$ Mobile Communication Technologies Conference, Oct. 2004.

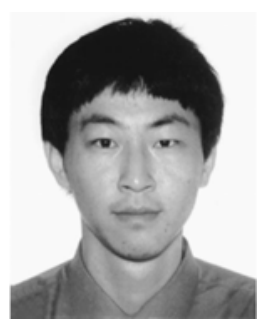

Tao Cui (S'04) received the M.Sc. degree in the Department of Electrical and Computer Engineering, University of Alberta, Edmonton, AB, Canada, in 2005, and the M.S. degree from the Department of Electrical Engineering, California Institute of Technology, Pasadena, USA, in 2006. He is currently working toward the Ph.D. degree at the Department of Electrical Engineering, California Institute of Technology, Pasadena. His research interests are in the interactions between networking theory, communication theory, and information theory.

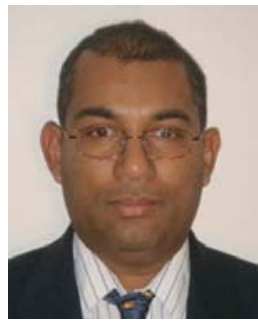

Chintha Tellambura (SM'02) received the B.Sc. degree (with first-class honors) from the University of Moratuwa, Moratuwa, Sri Lanka, in 1986, the M.Sc. degree in electronics from the University of London, London, U.K., in 1988, and the $\mathrm{Ph} . \mathrm{D}$. degree in electrical engineering from the University of Victoria, Victoria, BC, Canada, in 1993.

He was a Postdoctoral Research Fellow with the University of Victoria (1993-1994) and the University of Bradford (1995-1996). He was with Monash University, Melbourne, Australia, from 1997 to 2002. Presently, he is a Professor with the Department of Electrical and Computer Engineering, University of Alberta. His research interests include Diversity and Fading Countermeasures, Multiple-Input Multiple-Output (MIMO) Systems and Space-Time Coding, and Orthogonal Frequency Division Multiplexing (OFDM).

Prof. Tellambura is an Associate Editor for the IEEE Transactions on Communications and the Area Editor for Wireless Communications Systems and Theory in the IEEE Transactions on Wireless Communications. He was Chair of the Communication Theory Symposium in Globecom'05 held in St. Louis, MO. 